\title{
Static Error Free Tracking Control Based on Linear Quadratic Regulator of 3-DOF Parallel Manipulator with Redundant Actuation
}

\author{
Zhou Jiehua $^{1, *}$ and Peng Xiafu ${ }^{2}$ \\ ${ }^{1}$ School of Information Engineering, Nanchang Hangkong University, Nanchang, 330063, China \\ ${ }^{2}$ College of Information \& Technology, Xiamen University, Xiamen, 361005, China
}

\begin{abstract}
Parallel manipulators have advantages such as high stiffness, low moving inertia, high payload capability, and high accuracy. In this paper, the servo drive system of valve controlled asymmetrical hydraulic cylinder is designed for 4SPS/S parallel manipulator with redundant actuator. A unified nonlinear model suitable for forward and inverse motion is deduced for the valve controlled asymmetrical cylinder system, and simplified from point of engineering application. The global linearized model is established by nonlinear state feedback transformation. The tracking controller with zero steady-state errors based on linear quadratic regulator is designed for the global linearized model. Simulation results show that tracking performance of the proposed controller is good.
\end{abstract}

Keywords: Parallel manipulator, static error free tracking control, linear quadratic regulator, redundant actuation.

\section{INTRODUCTION}

Since Stewart's proposal to employ a parallel manipulator as a flight simulator in 1965 [1], over the last three decades, parallel manipulators are widely used in the entertainment and machine tool industries, especially in motion simulators [2]. They have many distinct advantages compared with the serial manipulators, such as the small accumulated error, fast response speed, high system stiffness, and a higher force-to-weight ratio [3]. The control of parallel manipulator has attracted much attention over the last decade. A number of studies on the control of parallel manipulator have been published, such as Stefano and Lorenzo proposed a force/position control strategies to deal with tasks involving interaction with the environment [4]. Whitey thoroughly analyzed the control and stability issues of the closed-loop force control of a one-link manipulator [5]. Lu and James proposed adaptive synchronized control for a planar parallel manipulator [6]. Djaferis proposed a robust hybrid control algorithm which achieves result by choosing controller's gains that minimize a performance index at a given range of modelling errors [7], Hag, Young and Kyo proposed a robust nonlinear controller equipped with a friction estimator for a 6 degree of freedom parallel manipulator in the task space coordinates [8], and so on.

However, accurate trajectory tracking control for parallel manipulators is still a key system requirement, as these devices must often follow prescribed motion $[9,10]$. Tracking control of parallel manipulators has been approached using both linear and nonlinear control laws $[11,12]$. High performance control strategies can significantly improve the tracking performance $[3,12]$. The dynamics of hydraulic systems are highly nonlinear due to the phenomena such as fluid compressibility, nonlinear servo valve flow-pressure characteristics, and dead band due to the internal leakage and hysteresis [13-15]. However, compared with electrical actuators, hydraulic actuators have many distinct advantages such as the ability to produce large forces at high speeds, the high durability and stiffness, and rapid response $[13,15]$.

Therefore, this paper will address the tracking control of hydraulic parallel manipulator. The rest of this paper is organized as follows. In Section 2, we present the structure of 4SPS/S parallel manipulator with redundant actuator and hydraulic drive system. Then a unified nonlinear model suitable for forward and inverse motion is deduced for the valve controlled asymmetrical cylinder system in Section 3. Thereafter, the proposed method for static error free tracking control based on linear quadratic regulator $(\mathrm{LQR})$ is presented in Section 4. In Section 5, the trajectory tracking simulations are carried out. In the end, a short conclusion is drawn in Section 6 .

\section{SYSTEM OVERVIEW AND MODELLING}

The three degree-of-freedom (DOF) hydraulic parallel manipulator includes a moving platform, a fixed base platform, four spherical joint-prismatic joint-spherical joint (SPS) type active legs with the linear hydraulic actuators, and one spherical joint type constrained passive leg, see Fig. (1). Here, the constraint passive leg is perpendicular to the fixed base platform, and connects the moving platform with the fixed platform by a flange plate on the fixed base platform at $A_{5}$ and a spherical joint on the moving platform at 


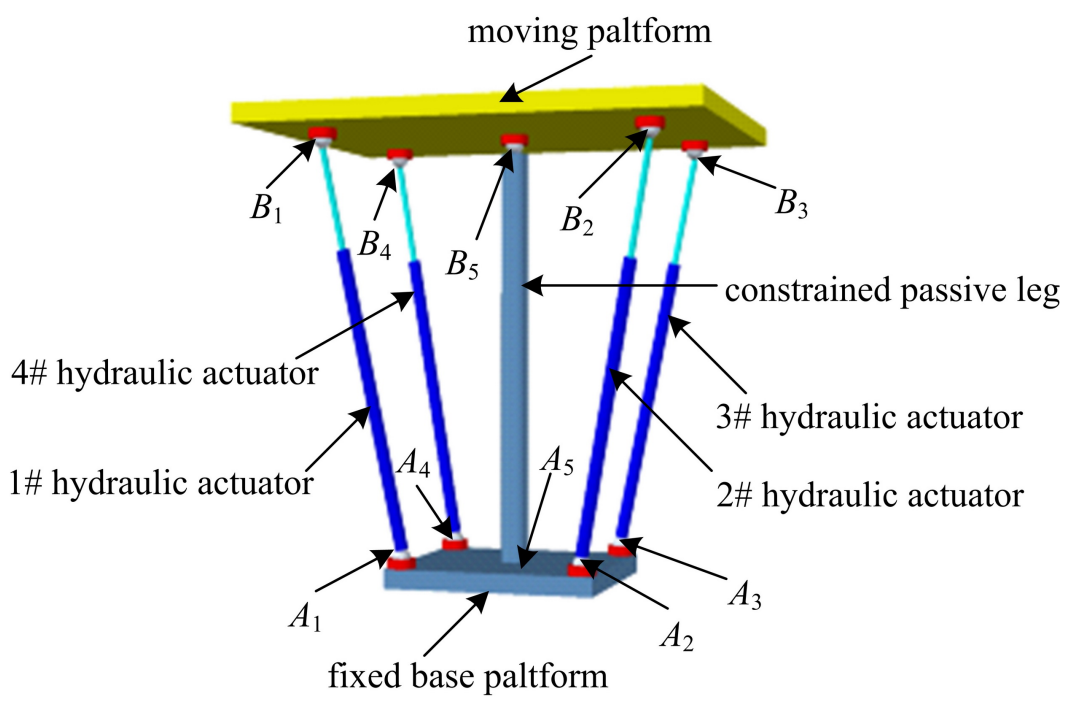

$$
\begin{gathered}
A_{1}, A_{2}, A_{3}, A_{4}, B_{1}, B_{2}, B_{3}, B_{4} \text { and } B_{5} \text { are spherical joints } \\
A_{5} \text { is flange plate }
\end{gathered}
$$

Fig. (1). Simplified 3-dof hydraulic parallel manipulator model.

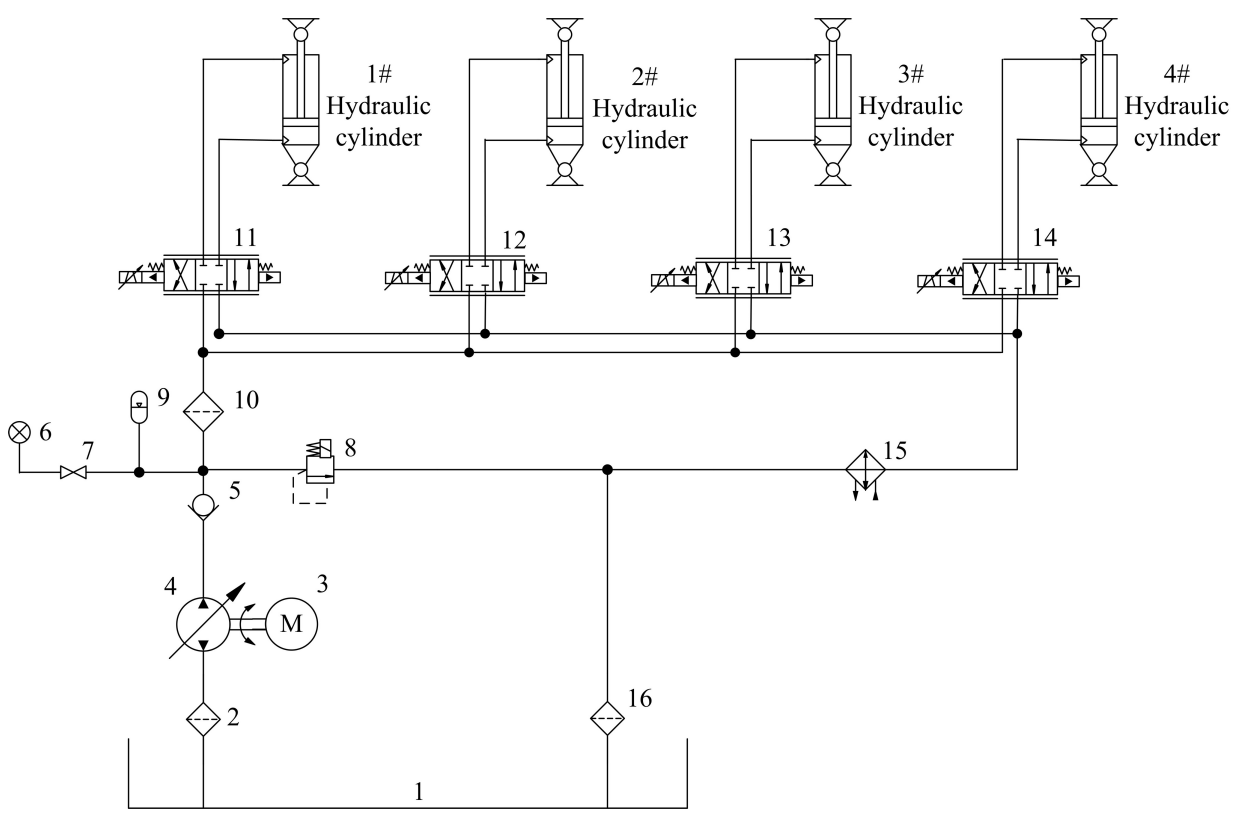

Fig. (2). Diagram of hydraulic drive system.

$B_{5}$. Each of the SPS-type active legs connects the moving platform to the fixed platform by a spherical joint at $B_{i}(i=1$, $2,3,4)$, an active leg with a prismatic joint, and a spherical joint at $A_{i}(i=1,2,3,4)$, respectively. Quadrilateral $B_{1} B_{2} B_{3} B_{4}$ is a rectangle with the side $B_{1} B_{2}=l_{1}, B_{2} B_{3}=l_{2}$, and a center point $B_{5}$. Quadrilateral $A_{1} A_{2} A_{3} A_{4}$ is also a rectangle with the side $A_{1} A_{2}=l_{3}, A_{2} A_{3}=l_{4}$, and a center point $A_{5}$. The length of constraint passive leg is $h$.

Fig. (2) shows the hydraulic drive system schematic diagram of 4-SPS/S three degree-of-freedom redundant actuated parallel, where element 1 is hydraulic oil tank, element 2 and 16 are crude oil filters, element 3 is motor, element 4 is vari- able displacement pump, element 5 is check valve, element 6 is pressure gauge, element 7 is pipe joint of the pressure gauge, element 8 is electronic control overflow valve, element 9 is accumulator, element 10 is fine oil filter, element $11,12,13$ and 14 are electro-hydraulic servo valves, element 15 is cooler.

\section{MATHEMATICAL MODELLING OF HYDRAULIC DRIVE SYSTEM}

Fig. (3) shows the diagram of hydraulic system with the asymmetric valve controlling asymmetric cylinder, where $y$ 


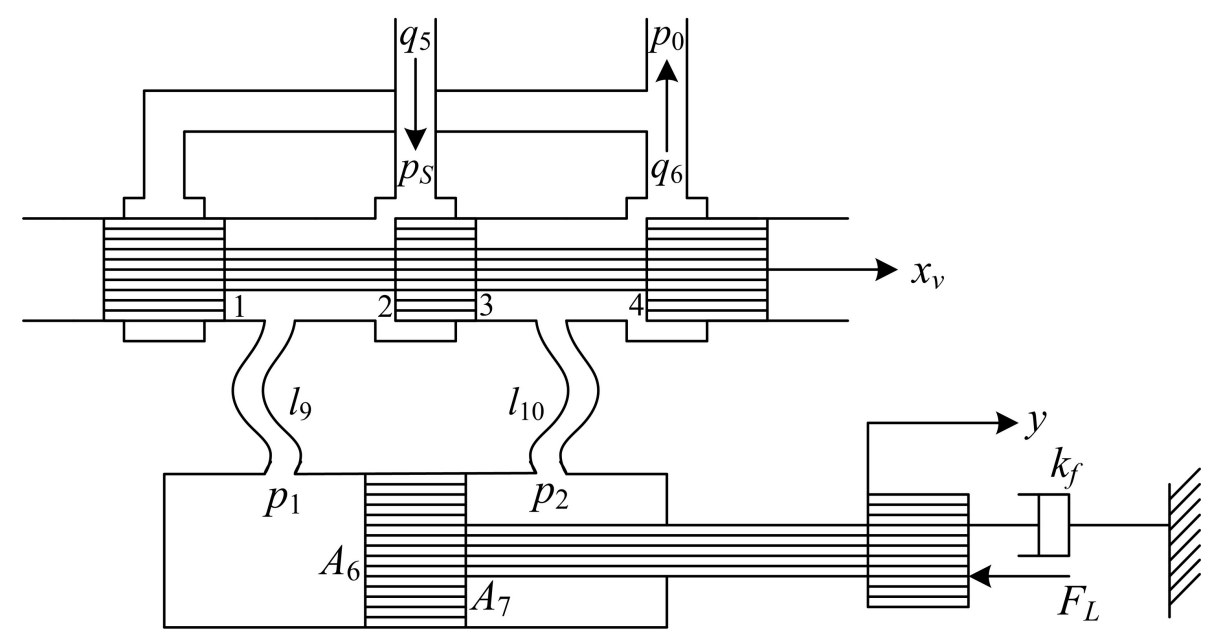

Fig. (3). Diagram of hydraulic system with valve controlled asymmetric cylinder.

is the piston rod displacement, $\dot{y}$ is the piston rod velocity, $\ddot{y}$ is the piston rod acceleration. $M_{a}$ is the total mass of the piston rod and load, $k_{f}$ is the viscosity damping coefficient, $F_{L}$ is the load force, $x_{v}$ is spool displacement. The oil inlet and return throttle window area-grads of servo valve are represented by $w_{1}, w_{2}, w_{3}$, and $w_{4}$, where $w_{1}$ and $w_{2}$ are areagrads of throttle window 1 and throttle window 2, respectively, connecting the servo valve and the hydraulic cylinder norod chamber, $w_{3}$ and $w_{4}$ are area-grads of throttle window 3 and throttle window4 connecting the servo valve and the hydraulic cylinder rod chamber. $p_{s}$ and $p_{0}$ are supply pressure and spill pressure of system. $p_{1}, p_{2}, q_{5}$ and $q_{6}$ are pressure and flow of rod chamber and no-rod chamber. $A_{6}, A_{7}, V_{01}, V_{02}, V_{1}$ and $V_{2}$ are effective area, initial volume and volume of rod chamber and no-rod chamber. $C_{i}$ and $C_{e}$ are internal leakage coefficient and external leakage coefficient of hydraulic cylinder. $C_{d}$ is flow coefficient. $\rho$ is density of hydraulic oil. $E_{5}$ is bulk modulus of hydraulic oil. $l_{9}$ and $l_{10}$ are the lengths of flexible pipe connecting the rod chamber with no-rod chamber. $D_{1}, h_{3}$ and $E_{6}$ are diameter, wall thickness elastic coefficient of flexible pipe. Directional variables are positive direction shown in the Fig. (3).

Assume that the supply pressure $p_{s}$ is stable and the spill pressure $p_{0}=0$ in the work process. The oil inlet and return throttle window area-grads of servo valve meet the follow conditions: $w_{1}=w_{2}, w_{3}=w_{4}$ and $\frac{w_{3}}{w_{1}}=\frac{w_{4}}{w_{2}}=n_{0}$, where, $0<n_{0} \leq 1$. The effective area of rod chamber and no-rod chamber meet the following condition: $\frac{A_{7}}{A_{6}}=m_{0}, 0<m_{0} \leq 1$. The $\operatorname{sgn}(\partial)$ is a sign function, $\partial>0, \operatorname{sgn}(\partial)=1 ; \partial>0$, $\operatorname{sgn}(\partial)=-1$.

The pressure-flow equation of slide valve is:

$q_{5}=C_{d} w_{1} x_{v} \sqrt{\frac{1+\operatorname{sgn}\left(x_{v}\right)}{\rho}\left(p_{s}-p_{1}\right)+\frac{1-\operatorname{sgn}\left(x_{v}\right)}{\rho} p_{1}}$,
$q_{6}=C_{d} w_{3} x_{v} \sqrt{\frac{1-\operatorname{sgn}\left(x_{v}\right)}{\rho}\left(p_{s}-p_{2}\right)+\frac{1+\operatorname{sgn}\left(x_{v}\right)}{\rho} p_{2}}$.

The flow continuity equation of hydraulic cylinder is:

$q_{5}=A_{6} \frac{\mathrm{d} y}{\mathrm{~d} t}+C_{i}\left(p_{1}-p_{2}\right)+C_{e} p_{1}+\xi_{1} \frac{\mathrm{d} p_{1}}{\mathrm{~d} t}$,

$q_{6}=A_{7} \frac{\mathrm{d} y}{\mathrm{~d} t}+C_{i}\left(p_{1}-p_{2}\right)-C_{e} p_{2}-\xi_{2} \frac{\mathrm{d} p_{2}}{\mathrm{~d} t}$,

Where,

$\xi_{1}=\frac{V_{01}+A_{6} y}{E_{5}}+\frac{l_{9} \pi D_{1}^{2}}{4}\left(\frac{1}{E_{5}}+\frac{D_{1}}{h_{3} E_{6}}\right)$,

$\xi_{2}=\frac{V_{02}-A_{7} y}{E_{5}}+\frac{l_{10} \pi D_{1}^{2}}{4}\left(\frac{1}{E_{5}}+\frac{D_{1}}{h_{3} E_{6}}\right)$.

The equilibrium equation of hydraulic cylinder is:

$A_{6} p_{1}-A_{7} p_{2}-k_{f} \frac{\mathrm{d} y}{\mathrm{~d} t}-F_{L}=M_{a} \frac{\mathrm{d}^{2} y}{\mathrm{~d} t}$.

System state variables are defined as: $\mathbf{x}=\left[\begin{array}{llll}x_{1} & x_{2} & x_{3} & x_{4}\end{array}\right]^{\mathrm{T}}=\left[\begin{array}{llll}y & \dot{y} & p_{1} & p_{2}\end{array}\right]^{\mathrm{T}}, \quad$ system input and output variables are defined as $u=x_{v}$ and $y$. Synthesizing equation (1) to equation (5), the state space model of hydraulic drive system is:

$$
\left\{\begin{array}{l}
\dot{x}_{1}=x_{2} \\
\dot{x}_{2}=\frac{A_{6}}{M_{a}} x_{3}-\frac{A_{7}}{M_{a}} x_{4}-\frac{k_{f}}{M_{a}} x_{2}-\frac{F_{L}}{M_{a}} \\
\dot{x}_{3}=g_{1}(\mathbf{x}) u-\frac{A_{6}}{\lambda_{1}+\lambda_{2} x_{1}} x_{2}-\xi_{3} \\
\dot{x}_{4}=g_{2}(\mathbf{x}) u+\frac{A_{7}}{\lambda_{3}+m_{0} \lambda_{2} x_{1}} x_{2}+\xi_{4}
\end{array},\right.
$$


Where,

$g_{1}(\mathbf{x})=\frac{C_{d} w_{1}}{\lambda_{1}+\lambda_{2} x_{1}} \sqrt{\frac{1+\operatorname{sgn}(u)}{\rho}\left(p_{s}-x_{3}\right)+\frac{1-\operatorname{sgn}(u)}{\rho} x_{3}}$,

$g_{2}(\mathbf{x})=-\frac{C_{d} w_{3}}{\lambda_{3} m_{0} \lambda_{y}} \sqrt{\frac{1-\operatorname{sgn}(u)}{2}\left(p_{s}-x_{4}\right)+\frac{1+\operatorname{sgn}(u)}{\rho} x_{4}}$

$u=x_{v}, \lambda_{1}=\frac{\lambda_{3} D_{01}^{m_{0}} \lambda_{3} x_{\pi} D_{1}^{2}}{E_{5}}+\frac{1}{4}\left(\frac{1}{E_{5}}+\frac{D_{1}}{h_{3} E_{6}}\right), \lambda_{2}=\frac{A_{6}}{E_{5}}$,

$\lambda_{3}=\frac{V_{02}}{E_{5}}+\frac{l_{10} \pi D_{1}^{2}}{4}\left(\frac{1}{E_{5}}+\frac{D_{1}}{h_{3} E_{6}}\right)$,

$\xi_{3}=\frac{C_{i}}{\lambda_{1}+\lambda_{2} x_{1}}\left(x_{3}-x_{4}\right)-\frac{C_{e}}{\lambda_{1}+\lambda_{2} x_{1}} x_{3}$,

$\xi_{4}=\frac{C_{i}}{\lambda_{3}+m_{0} \lambda_{2} x_{1}}\left(x_{3}-x_{4}\right)-\frac{C_{e}}{\lambda_{3}+m_{0} \lambda_{2} x_{1}} x_{4}$

\section{HYDRAULIC DRIVE SYSTEM INPUT-OUTPUT FEEDBACK LINEARIZATION}

The servo valve is directly installed on the cylinder of hydraulic drive system, so the length of flexible pipe is very short, and it can be ignored in engineering. Furthermore,

because of $\frac{V_{01}}{E_{5}} \gg \frac{A_{6}}{E_{5}}$, the nonlinear state space equation (6) is transformed to affine nonlinear system, and its standard form is:

$\left\{\begin{array}{l}\dot{\mathbf{x}}=\mathbf{f}(\mathbf{x})+\mathbf{g}(\mathbf{x}) u+\mathbf{Q}_{1} \\ y=h(\mathbf{x})\end{array}\right.$

Where,

$\mathbf{f}(\mathbf{x})=\left[\begin{array}{c}x_{2} \\ \frac{A_{6}}{M_{a}} x_{3}-\frac{A_{7}}{M_{a}} x_{4}-\frac{k_{f}}{M_{a}} x_{2} \\ -\frac{A_{6}}{\lambda_{1}} x_{2}-\frac{C_{i}}{\lambda_{1}}\left(x_{3}-x_{4}\right)-\frac{C_{e}}{\lambda_{1}} x_{3} \\ \frac{A_{7}}{\lambda_{3}} x_{2}+\frac{C_{i}}{\lambda_{3}}\left(x_{3}-x_{4}\right)-\frac{C_{e}}{\lambda_{3}} x_{4}\end{array}\right]$,

$\mathbf{g}(\mathbf{x})=\left[\begin{array}{llll}0 & 0 & g_{1}(\mathbf{x}) & g_{2}(\mathbf{x})\end{array}\right]^{\mathrm{T}}, u=x_{v}, h(\mathbf{x})=x_{1}$,

$\mathbf{Q}_{1}=\left[\begin{array}{llll}0 & -\frac{F_{L}}{M_{a}} & 0 & 0\end{array}\right]^{\mathrm{T}}$

By the equation (7) can be obtained:

$L_{\mathbf{f}} h(\mathbf{x})=\frac{\partial h}{\partial \mathbf{x}} \mathbf{f}(\mathbf{x})=x_{2}, L_{\mathbf{g}} h(\mathbf{x})=\frac{\partial h}{\partial \mathbf{x}} \mathbf{g}(\mathbf{x})=0, L_{\mathbf{g}} L_{\mathbf{f}} h(\mathbf{x})=0$,

$$
\begin{aligned}
& L_{\mathbf{f}}^{2} h(\mathbf{x})=\frac{A_{6}}{M_{a}} x_{3}-\frac{A_{7}}{M_{a}} x_{4}-\frac{k_{f}}{M_{a}} x_{2}, \\
& L_{\mathbf{g}} L_{\mathbf{f}}^{2} h(\mathbf{x})=\frac{A_{6}}{M_{a}} g_{1}(\mathbf{x})-\frac{A_{7}}{M_{a}} g_{2}(\mathbf{x}), \\
& L_{\mathbf{f}}^{3} h(\mathbf{x})=-\frac{k_{f}}{M_{a}^{2}}\left(A_{6} x_{3}-A_{7} x_{4}-B_{c} x_{2}\right)-\xi_{5}-\xi_{6}, \\
& \xi_{5}=\frac{A_{6}}{M_{a} \lambda_{1}}\left(A_{6} x_{2}+C_{i}\left(x_{3}-x_{4}\right)+C_{e} x_{3}\right), \\
& \xi_{6}=\frac{A_{7}}{M_{a} \lambda_{3}}\left(A_{7} x_{2}+C_{i}\left(x_{3}-x_{4}\right)-C_{e} x_{4}\right) .
\end{aligned}
$$

Select the coordinate transformation is:

$$
\mathbf{z}=\Phi(\mathbf{x})=\left[\begin{array}{c}
\phi_{1}(\mathbf{x}) \\
\phi_{2}(\mathbf{x}) \\
\phi_{3}(\mathbf{x}) \\
\phi_{4}(\mathbf{x})
\end{array}\right]=\left[\begin{array}{c}
h(\mathbf{x}) \\
L_{\mathbf{f}} h(\mathbf{x}) \\
L_{\mathbf{f}}^{2} h(\mathbf{x}) \\
\eta(\mathbf{x})
\end{array}\right] .
$$

According to the reference [16], a function $\eta(\mathbf{x})$ which makes the map $\Phi(\mathbf{x})$ non-singular Jacobian matrix at $\mathrm{x}_{0}$ can be found, and $\eta(\mathbf{x})$ can be selected as:

$L_{\mathbf{g}} \eta(\mathbf{x})=\frac{\partial \eta}{\partial \mathbf{x}} \mathbf{g}(\mathbf{x})=0$

So, the equation (9) can be transformed:

$$
\frac{\partial \eta}{\partial \mathbf{x}} \mathbf{g}(\mathbf{x})=\frac{\partial \eta}{\partial x_{3}} g_{1}(\mathbf{x})+\frac{\partial \eta}{\partial x_{4}} g_{2}(\mathbf{x})=0 \text {. }
$$

The equation (11) is a solution of equation (10):

$$
\eta(\mathbf{x})=\lambda_{1} w_{3} \sqrt{[1+\operatorname{sgn}(u)]\left(p_{s}-x_{3}\right)+[1-\operatorname{sgn}(u)] x_{3}}+\xi_{7},
$$

Where,

$$
\xi_{7}=\lambda_{3} w_{1} \sqrt{[1-\operatorname{sgn}(u)]\left(p_{s}-x_{4}\right)+[1+\operatorname{sgn}(u)] x_{4}} \text {. }
$$

After calculation shows Jacobian matrix of $\Phi(\mathbf{x})$ is nonsingular, so the transformation of equation (8) is a global differential homeomorphism, and the original system is transformed as:

$\left\{\begin{array}{l}\dot{\mathbf{z}}=\left[\begin{array}{lll}0 & 1 & 0 \\ 0 & 0 & 1 \\ 0 & 0 & 0\end{array}\right]\left[\begin{array}{c}z_{1} \\ z_{2} \\ z_{3}\end{array}\right]+\left[\begin{array}{l}0 \\ 0 \\ 1\end{array}\right] v+\tilde{\mathbf{Q}}_{1}, \\ y=z_{1}\end{array}\right.$

Where, 


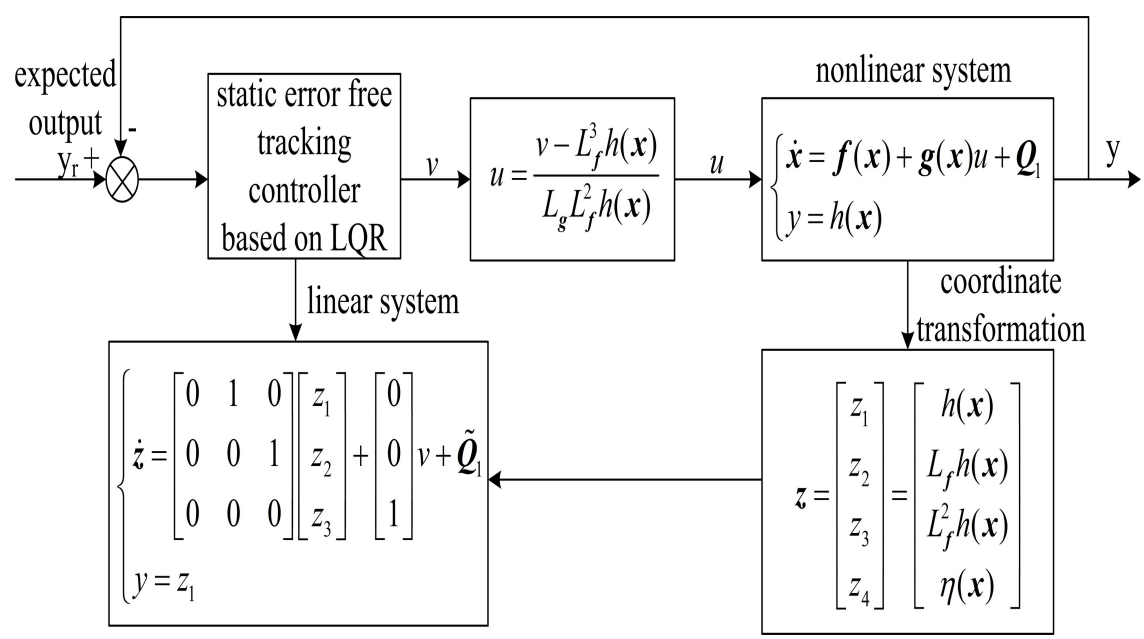

Fig. (4). Scheme of control system.

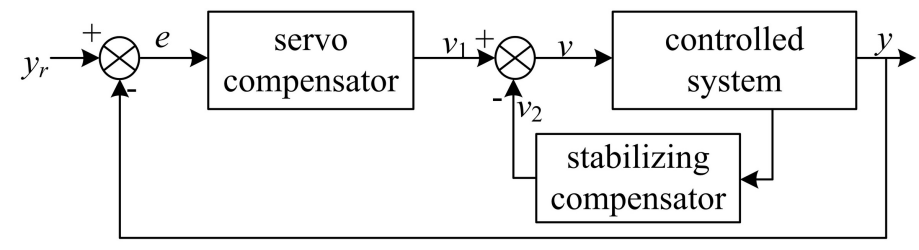

(a) General composition structure

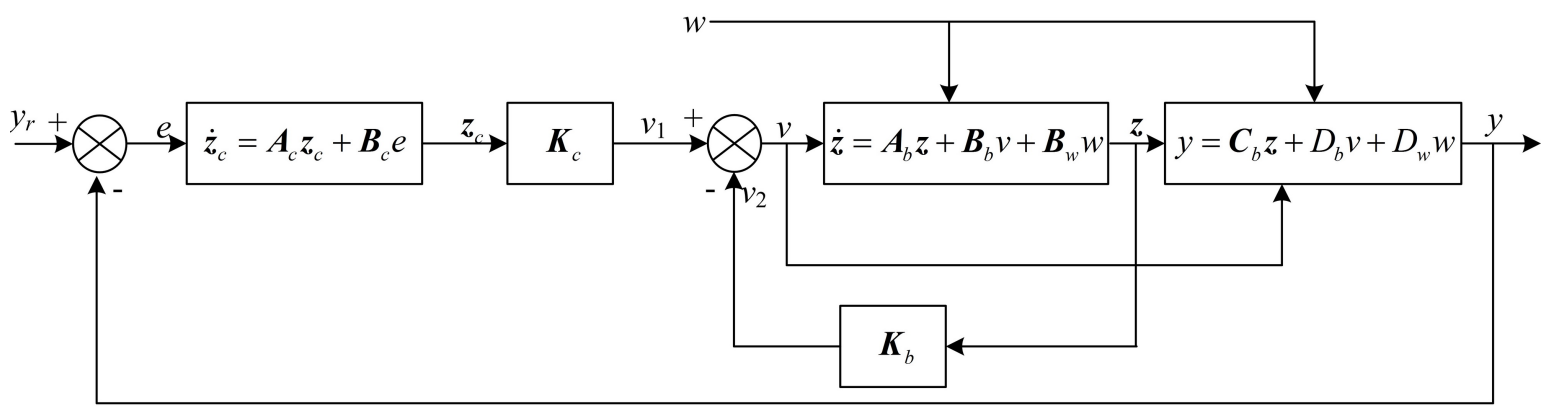

(b) Detailed composition structure

Fig. (5). Static error free tracking control system.

$\tilde{\mathbf{Q}}_{1}=\left[\begin{array}{lll}0 & -\frac{F_{L}}{M_{a}} & \frac{k_{f} F_{L}}{M_{a}^{2}}\end{array}\right]^{\mathrm{T}}, v=L_{\mathbf{f}}^{3} h(\mathbf{x})+L_{\mathbf{g}} L_{\mathbf{f}}^{2} h(\mathbf{x}) u$.

According to controllability criterion of linear system, the linear system of equation (12) is completely controllable. The equation (12) represents the external state of system, but the internal state $\eta$ becomes unobservable because of state feedback. For precise input-output linearization system, the external state should be stable and have a good dynamic quality, and the internal state $\eta$ should also be made stable as well. According to the reference [16] analysis, the internal state $\eta$ of system is stable.

The state space model of hydraulic drive system can be transformed into the linear system of equation (12) by feedback linearization method, so the controller can be designed by linear system method. In this paper, the static error free tracking control based on LQR is used to control hydraulic drive system, and the structure of control system is shown in Fig. (4). The general composition structure of static error free tracking control is shown in Fig. (5a), and the controller consists of servo compensator and stabilizing compensator. The servo compensator is a linear time invariant system, and its function is to control the system to achieve asymptotic tracking and disturbance rejection. The stabilizing compensator is a static state feedback, and its function is to make the control system achieve asymptotical stability. Fig. (5b) is the detailed composition structure of static error free tracking control.

Let the controlled system be completely controlled, and its state space is described as: 


$$
\left\{\begin{array}{l}
\dot{\mathbf{z}}=\mathbf{A}_{b} \mathbf{z}+\mathbf{B}_{b} v+\mathbf{B}_{w} w \\
y=\mathbf{C}_{b} \mathbf{z}+D_{b} v+D_{w} w
\end{array}\right.
$$

Where, $n=\operatorname{dim}(\mathbf{z}), p=\operatorname{dim}(v), q=\operatorname{dim}(y)$ and $w$ is disturbance signal. The specific composition structure of static error free tracking control is shown in Fig. (5b), and the tracking error is $e(t)=y_{r}(t)-y(t)$.

The static error free tracking control algorithm based on LQR is as follows:

Step 1. Judge the relationship between the input dimensions and the output dimensions of controlled system, and analyze the controllability of controlled system. Furthermore, the input and output satisfy the following formula: $\operatorname{dim}(v) \geq \operatorname{dim}(y)$.

Step 2 . Derive the common instability polynomial $\phi(s)$ of $y_{r}(t)$ and $w(t)$, i.e. when $t \rightarrow \infty$, derive the frequency domain structure characteristics $\phi_{r}(s)$ and $\phi_{w}(s)$ by no zero parts $y_{r s}(t)$ and $w_{s}(t)$, and calculate the minimal common multiple $\phi(s)$ between $\phi_{r}(s)$ and $\phi_{w}(s), \quad$ and $\phi(s)=s^{l}+\tilde{\alpha}_{l-1} s^{l-1}+\cdots+\tilde{\alpha}_{1} s+\tilde{\alpha}_{0}$.

Step 3. Judge the rank relationship which can realize static error free tracking control, i.e. each root $\lambda_{i}$ of $\phi(s)=0$ can satisfy the following formula:

$\operatorname{rank}\left[\begin{array}{cc}\lambda_{i} \mathbf{I}-\mathbf{A}_{b} & \mathbf{B}_{b} \\ -\mathbf{C}_{b} & \mathbf{D}_{b}\end{array}\right]=n+q, i=1,2, \cdots, l$.

Step 4. Derive the block coefficient matrix:

$\Gamma=\left[\begin{array}{cccc}0 & & & \\ \vdots & & \mathbf{I}_{l-1} & \\ 0 & & & \\ -\tilde{\alpha}_{0} & -\tilde{\alpha}_{1} & \cdots & -\tilde{\alpha}_{l-1}\end{array}\right], \underset{l \times 1}{\beta}=\geq\left[\begin{array}{c}0 \\ \vdots \\ 0 \\ 1\end{array}\right]$,

And derive the common instability model $y_{r}(t)$ and $w(t)$ :

$\dot{\mathbf{z}}_{c}=\mathbf{A}_{c} \mathbf{z}_{c}+\mathbf{B}_{c} e$,

Where, $\underset{q \mid \times a l}{\mathbf{A}_{c}}=\left[\begin{array}{ccc}\Gamma & & \\ & 0 & \\ & & \Gamma\end{array}\right], \underset{q l \times q l}{\mathbf{B}_{c}}=\left[\begin{array}{lll}\beta & & \\ & 0 & \\ & & \beta\end{array}\right]$.

Step 5. Build the state equation of $(n+q l)$ dimensional series system $\Sigma_{T}$ :

$$
\begin{aligned}
& {\left[\begin{array}{c}
\dot{\mathbf{z}} \\
\dot{\mathbf{z}}_{c}
\end{array}\right]=\left[\begin{array}{cc}
\mathbf{A}_{b} & 0 \\
-\mathbf{B}_{c} \mathbf{C}_{b} & \mathbf{A}_{b}
\end{array}\right]\left[\begin{array}{c}
\mathbf{z} \\
\mathbf{z}_{c}
\end{array}\right]+\left[\begin{array}{c}
\mathbf{B}_{b} \\
-\mathbf{B}_{c} \mathbf{D}_{b}
\end{array}\right] .} \\
& v+\left[\begin{array}{c}
\mathbf{B}_{w} \\
-\mathbf{B}_{c} \mathbf{D}_{w}
\end{array}\right] w+\left[\begin{array}{c}
0 \\
\mathbf{B}_{c}
\end{array}\right] y_{r}
\end{aligned}
$$

Step 6. Establish $p \times(n+q l)$ dimensional matrix $\mathbf{K}_{T}$ based on LQR method.

Step7. Matrix $\mathbf{K}_{T}$ partition is the following formula:

$\mathbf{K}_{T}=\left[\begin{array}{cc}-\mathbf{K}_{b} & \mathbf{K}_{c} \\ p \times n & p \times q l\end{array}\right]$.

Step 8. Derive the stabilizing compensator: $v_{2}=\mathbf{K}_{b} \mathbf{z}$.

Step 9. Derive the servo compensator:

$\dot{\mathbf{z}}_{c}=\mathbf{A}_{c} \mathbf{z}_{c}+\mathbf{B}_{c} e, v_{1}=\mathbf{K}_{c} \mathbf{z}_{c}$.

The static error free tracking control algorithm based on LQR is applied to 3-DOF parallel manipulator with redundant actuation for trajectory tracking control, and the control strategy is shown in Fig. (6). Each active branch with load force compensation calculation model adopts the static error free tracking control algorithm based on LQR to implement control, where the closed loop control system is composed of the inverse position as desired trajectory of the hydraulic cylinder and the displacement sensor mounted inside the hydraulic cylinder as position feedback signal.

\section{SIMULATION RESULTS}

The design parameters of the parallel manipulator and hydraulic system are as follows: $l_{1}=2.4 \mathrm{~m}, l_{2}=3.6 \mathrm{~m}, l_{3}=1.8 \mathrm{~m}$, $l_{4}=2.0 \mathrm{~m}, h=4.7 \mathrm{~m}, w_{1}=w_{2}=3.76 \times 10^{-2} \mathrm{~m}, w_{3}=w_{4}=1.88 \times 10^{-2} \mathrm{~m}$, $k_{f}=850 \mathrm{~N} /(\mathrm{m} / \mathrm{s}), \quad A_{6}=6.362 \times 10^{-3} \mathrm{~m}^{2}, \quad A_{7}=3.244 \times 10^{-3} \mathrm{~m}^{2}$, $V_{01}=1.145 \times 10^{-2} \mathrm{~m}^{3}, \quad V_{02}=5.893 \times 10^{-2} \mathrm{~m}^{3}, \quad C_{i}=2.836 \times 10^{-}$ ${ }^{13} \mathrm{~m} /(\mathrm{s} . P a), \quad C_{e}=7.654 \times 10^{-13} \mathrm{~m} /(\mathrm{s} . P a), \quad C_{d}=0.67, \rho=875 \mathrm{~kg} / \mathrm{m}^{3}$, and $E_{5}=750 \mathrm{MPa}$. Since the structure of hydraulic drive system and controller are same, so $1 \#$ active branched chain is selected for experiment in order to analyze the effect of the controller. The load force can be calculated by dynamics, it gets the load force $F_{L} \quad[-6000 \mathrm{~N}, 6000 \mathrm{~N}]$, so the load force compensation model is selected, $F_{L}=6000 \sin \left(\frac{\pi}{4} t\right)$.

The control system corresponding to equation (13) is as follow:

$$
\begin{aligned}
\mathbf{A}_{b} & =\left[\begin{array}{lll}
0 & 1 & 0 \\
0 & 0 & 1 \\
0 & 0 & 0
\end{array}\right], \mathbf{B}_{b}=\left[\begin{array}{l}
0 \\
0 \\
1
\end{array}\right], \mathbf{B}_{w}=\left[\begin{array}{c}
0 \\
-1 / M_{a} \\
k_{f} / M_{a}^{2}
\end{array}\right], w=F_{L}, \\
\mathbf{C}_{b} & =\left[\begin{array}{l}
1 \\
0 \\
0
\end{array}\right]^{\mathrm{T}}, D_{b}=D_{w}=0 .
\end{aligned}
$$

So, the input and output of control system satisfy the following formula: $\operatorname{dim}(v) \geq \operatorname{dim}(y)$, and it is completely controllable. It has two roots $\lambda_{1}=-\frac{\pi}{4} j$ and $\lambda_{2}=\frac{\pi}{4} j$ for the $\phi(s)=0$, and the following result can be obtained by calculation: 


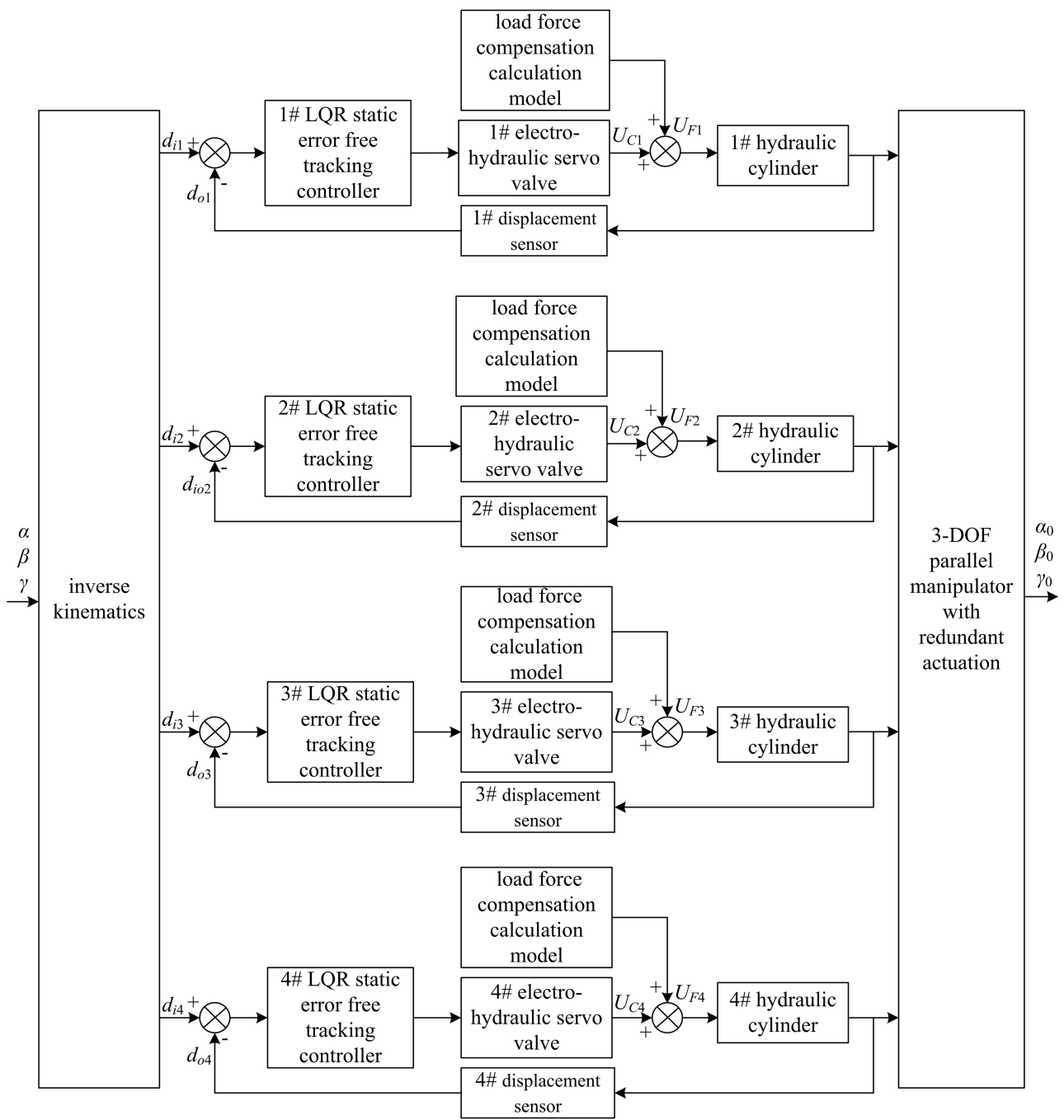

Fig. (6). Control strategy of 3-DOF parallel manipulator with redundant actuation.

$\operatorname{rank}\left[\begin{array}{cc}\lambda_{1} \mathbf{I}-\mathbf{A}_{b} & \mathbf{B}_{b} \\ -\mathbf{C}_{b} & \mathbf{D}_{b}\end{array}\right]=\operatorname{rank}\left[\begin{array}{cc}\lambda_{2} \mathbf{I}-\mathbf{A}_{b} & \mathbf{B}_{b} \\ -\mathbf{C}_{b} & \mathbf{D}_{b}\end{array}\right]=4=n+q$

Then it can satisfy the rank relationship meeting static error free tracking control.

The common instability model of $y_{r}(t)$ and $w(t)$ is:

$\dot{\mathbf{z}}_{c}=\mathbf{A}_{c} \mathbf{z}_{c}+\mathbf{B}_{c} e=\left[\begin{array}{cc}0 & 1 \\ -\frac{\pi^{2}}{16} & 0\end{array}\right] \mathbf{z}_{c}+\left[\begin{array}{l}0 \\ 1\end{array}\right] e$.

The state equation of $(n+q l)$ dimensional series system $\Sigma_{T}$ is:

$\left[\begin{array}{c}\dot{\mathbf{z}} \\ \dot{\mathbf{z}}_{c}\end{array}\right]=\left[\begin{array}{cc}\mathbf{A}_{b} & 0 \\ -\mathbf{B}_{c} \mathbf{C}_{b} & \mathbf{A}_{c}\end{array}\right]\left[\begin{array}{c}\mathbf{z} \\ \mathbf{z}_{c}\end{array}\right]+\left[\begin{array}{c}\mathbf{B}_{b} \\ -\mathbf{B}_{c} \mathbf{D}_{b}\end{array}\right] v$ $+\left[\begin{array}{c}\mathbf{B}_{w} \\ -\mathbf{B}_{c} \mathbf{D}_{w}\end{array}\right] w+\left[\begin{array}{c}0 \\ \mathbf{B}_{c}\end{array}\right] y_{r}$
The matrix values are substituted into the equation (14), by finishing can be as follow:

$$
\begin{aligned}
& {\left[\begin{array}{c}
\dot{\mathbf{z}} \\
\dot{\mathbf{z}}_{c}
\end{array}\right]=\left[\begin{array}{ccccc}
0 & 1 & 0 & 0 & 0 \\
0 & 0 & 1 & 0 & 0 \\
0 & 0 & 0 & 0 & 0 \\
0 & 0 & 0 & 0 & 1 \\
-1 & 0 & 0 & -\frac{\pi^{2}}{16} & 0
\end{array}\right]\left[\begin{array}{c}
\mathbf{z} \\
\mathbf{z}_{c}
\end{array}\right]+\left[\begin{array}{l}
0 \\
0 \\
1 \\
0 \\
0
\end{array}\right] .} \\
& v+\left[\begin{array}{c}
0 \\
-1 / M_{a} \\
k_{f} / M_{a}^{2} \\
0 \\
0
\end{array}\right] F_{L}+\left[\begin{array}{c}
0 \\
0 \\
0 \\
0 \\
1
\end{array}\right] y_{r}
\end{aligned}
$$

Easy to determine the system (15) is controllable, and apply LQR method to calculate the whole state feedback gain. It selects the weighting matrix $\mathrm{R}_{\mathrm{T}}=[1]$ and $\mathrm{Q}_{\mathrm{T}}=\operatorname{diag}([2$ 


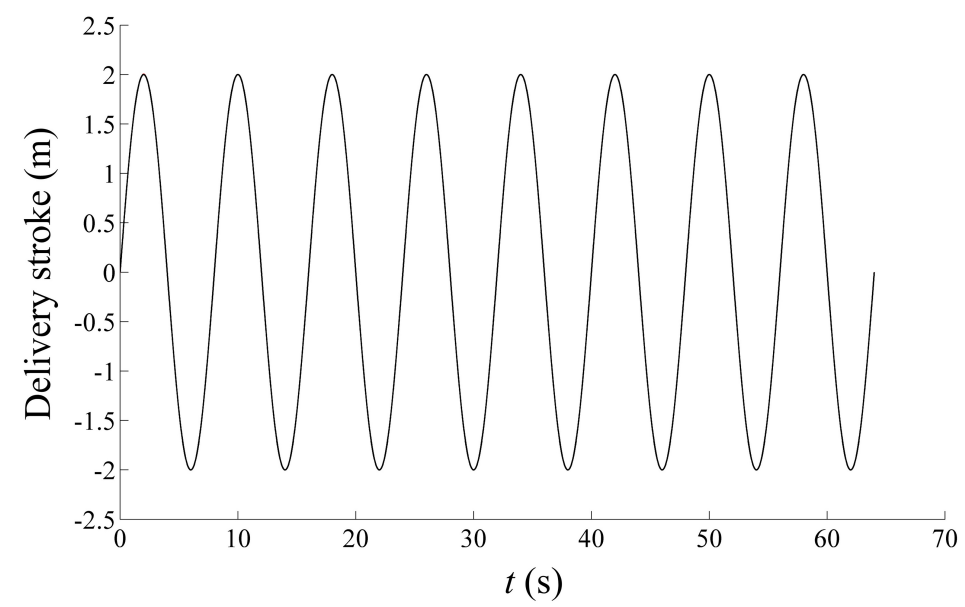

Fig. (7). Stroke tracking curve.

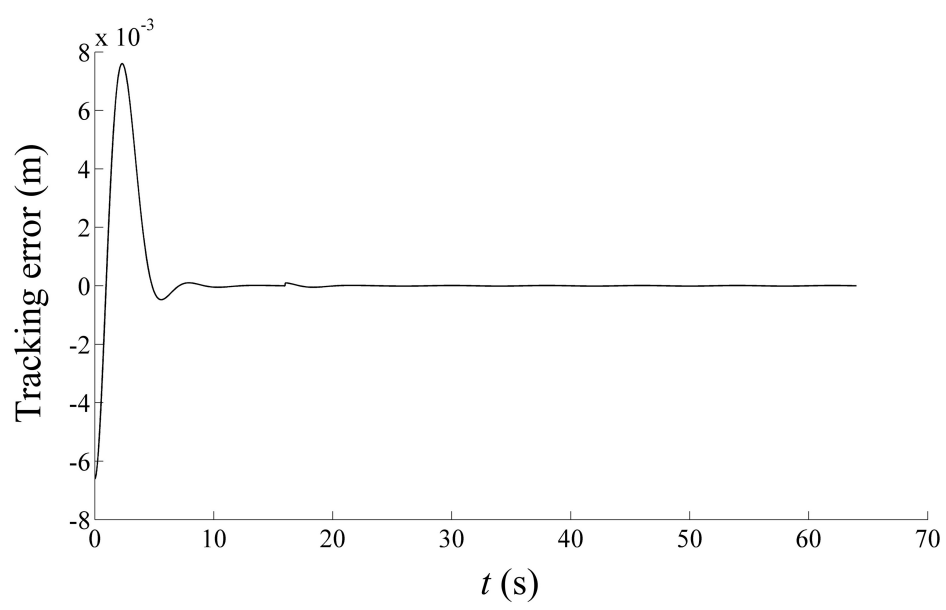

Fig. (8). Tracking error curve.

$\left.\left.\begin{array}{lllll}0 & 1 & 1 & 4\end{array}\right]\right)$, then the whole state feedback gain can be calculated by calling the function $\operatorname{lqr}($ ) of Matlab, it can obtain the whole state feedback gain $\mathrm{K}_{\mathrm{T}}=\left[\begin{array}{lll}13.8003 & 12.3432 & 5.8895\end{array}\right.$ $4.3163-5.0999]$. Simulation studies are conducted on Matlab-Simulink and $1 \#$ active branched input stroke is $y_{r}=2 \sin \left(\frac{\pi}{4} t\right)$. Fig. (7) shows the results of tracking $1 \#$ active branched input stroke and Fig. (8) shows the tracking error.

\section{CONCLUSION}

Hydraulic servo drive system has been designed for 4SPS/S 3-DOF parallel manipulator with redundant actuation. A unified nonlinear model has been theoretically established for forward and inverse motion of hydraulic cylinder, and the nonlinear model has been simplified from the perspective of engineering applications. On the basis of differential geometry linearization method, the global linear model is achieved by nonlinear state feedback transforming. Thereafter, on the basis of load force compensation, the static error free tracking controller based on LQR is designed. Simulations demonstrated the effectiveness of the proposed controller, and it can provide a theoretical basis for subsequent practical engineering implementation.

\section{CONFLICT OF INTEREST}

The author confirms that this article content has no conflict of interest.

\section{ACKNOWLEDGEMENTS}

The authors would like to express financial supports from Foundation Project of Jiangxi Provincial Department of Education (GJJ14549) and Doctor Scientific Research Foundation of Nanchang Hangkong University (EA201204428).

\section{REFERENCES}

[1] D. Stewart, "A platform with six degrees of freedom," Proceedings of the Institution of Mechanical Engineers, vol. 180, no. 15, pp. 371-386, 1965 
[2] Y. Pi and X. Wang, "Trajectory tracking control of a 6-dof hydraulic parallel robot manipulator with uncertain load disturbances", Control Engineering Practice, vol. 19, no. 2, pp. 185-193, 2011.

[3] B. Dasgupta and T.S. Mruthyunjaya, "The stewart platform manipulator: a review", Mechanism and Machine Theory, vol. 35, no. 1, pp. 15-40, 2000.

[4] S. Chiaverini and L. Sciavicco, "The parallel approach to force/position control of robotic manipulators", IEEE Transactions on Robotics and Automation, vol. 9, no. 4, pp. 361-373, 1993.

[5] D.E. Whitney, "Force feedback control of manipulator fine motion", ASME Journal of Dynamics Systems Measurement and Control, vol. 99, no. 2, pp. 91-97, 1977.

[6] L. Ren, J.K. Mills and D. Sun, "Adaptive synchronized control for a planar parallel manipulator: theory and experiments", ASME Journal of Dynamic Systems Measurement and Control, vol. 128, no. 4, pp. 976-979, 2006.

[7] T.E. Djaferis, B. Murah and J. Franklin. "Compliant control using robust multivariable feedback methods," Proceedings of American Control Conference 2021-2026, 1987.

[8] H.S. Kim, Y.M. Cho and K. Lee, "Robust nonlinear task space control for 6 DOF parallel manipulator", Automatica, vol. 41, no. 9, pp. 1591-1600, 2005.

[9] S. Tafazoli, C.W. de Silva and P.D. Lawrence, "Tracking control of an electrohydraulic manipulator in the presence of friction", IEEE Transaction on Control Systems Technology, vol. 6, no. 3, pp. 401411, 1998
[10] A. Ghobakhloo, M. Eghtesad and M. Azadi, "Position control of a stewart-gough platform using inverse dynamics method with full dynamics," $9^{\text {th }}$ IEEE International Workshop on Advanced Motion Control, Istanbul, Turkey, pp. 50-55, 2006.

[11] G.A. Sohl and J.E. Bobrow, "Experiments and simulations on the nonlinear control of a hydraulic servosystem", IEEE Transactions on Control Systems Technology, vol. 7, no. 2, pp. 238-247, 1999.

[12] M.R. Sirouspour and S.E. Salcudean, "Nonlinear control of hydraulic robots", IEEE Transactions on Robotics and Automation, vol. 17, no. 2, pp. 173-182, 2001.

[13] B. Yao, F.P. Bu, J. Reedy, G.T.-C Chiu, "Adaptive robust motion control of single-rod hydraulic actuators: theory and experiments", IEEE/ASME Transactions on Mechatronics, vol. 5, no. 1, pp. 7991, 2000.

[14] W.H. Zhu and J.C. Piedboeuf, "Adaptive output force tracking control of hydraulic cylinders with applications to robot manipulators", Journal of Dynamic Systems Measurement and Control Transactions of the ASME, vol. 127, no. 2, pp. 206-217, 2005.

[15] H.M Chen, J.C. Ren and J.P Su, "Sliding mode control with varying boundary layers for an electro-hydraulic position servo system", International Journal of Advanced Manufacturing Technology, vol. 26, no. 1, pp. 117-123, 2005.

[16] J. Yang, Z. Yin and S. Li, "Nonlinear modelling and feedback linearization of vavle-controlled asymmetrical cylinder", Chinese Journal of Mechanical Engineering, vol. 42, no. 5, pp. 203-207, 2006. (in Chinese)

Received: November 10, 2014

(C) Jiehua and Xiafu; Licensee Bentham Open.

This is an open access article licensed under the terms of the Creative Commons Attribution Non-Commercial License (http://creativecommons.org/licenses/by-nc/3.0/) which permits unrestricted, non-commercial use, distribution and reproduction in any medium, provided the work is properly cited. 